Journal of Data Science 4(2006), 21-37

\title{
A Dynamic Spatial Model for Chronic Wasting Disease in Colorado
}

\author{
Craig J. Johns and Christopher H. Mehl \\ University of Colorado
}

\begin{abstract}
A spatio-temporal statistical model for Chronic Wasting Disease is presented. The model has underpinnings from traditional epidemic models with differential equations and uses a Bayesian hierarchy to directly incorporate existing prevalence data. Spatial dynamics are modeled explicitly through a system of difference equations rather than through covariance. The posterior distribution gives evidence of a long term stable level of disease prevalence, and approximates the probability of the movement of the disease from one area to another. Predictions for the future of Chronic Wasting Disease in Colorado are given. The model is used to formulate efficient sampling schemes for future data collection.
\end{abstract}

Key words: Chronic wasting disease, data-model merge, dynamic models, spatio-temporal models.

\section{Introduction}

Chronic wasting disease (CWD) is a transmissible spongiform encephalopathy (TSE) that occurs in wild cervid populations in North America. It has been found in mule deer (Cervus odocoileus hemionus), white-tail deer (Cervus odocoileus virginianus) and elk (Cervus elaphus nelsoni) see Miller et al., 2000, Gross and Miller, 2001. CWD is related to other TSE's found in domestic animals, such as scrapie in sheep and bovine spongiform encephalopathy, commonly called mad cow disease (Miller et al., 2000). It is also related to TSE's found in humans, such as kuru, classic Creutzfelt-Jacob disease, and variant Creutzfelt-Jacob disease. The agent that causes chronic wasting disease is related to a prion that affects nervous tissue, yet the method of transmission is currently unknown (Colorado 2003, Miller et al., 2000, Gross and Miller, 2001, Hobbs et al., 2002).

The course of infection in mule deer appears to include both latent and infectious periods, with a total span of between 18 and 36 months. CWD causes damage to portions of the brain in affected animals. Animals with CWD show a progressive loss of body condition, behavioral changes, and eventually death 
Colorado, 2003, Gross and Miller, 2001). Once clinical signs appear, few deer survive more than 12 months (Gross and Miller, 2001).

Thus far, chronic wasting disease has been far more common in deer than in elk. It has been estimated that in infected regions, estimates are in the 5-6\% range for mule deer, compared to only about 1\% in elk (Gross anf Miller, 2001, Colorado, 2003). While both mule deer and white-tailed deer can contract the disease, in the Rocky Mountain region the epidemic seems to be focused in those areas where mule deer are common and relatively few white-tailed deer are found. Consequently, research on the dynamics of the disease in the Rocky Mountain area (Gross and Miller, 2001, Hobbs et al. 2002, Hobbs et al., 2003) has focused on mule deer.

Because of the economic impact that hunting and related business has in some states, Chronic Wasting Disease is recognized throughout the United States as a potentially disastrous problem. Currently, every state except Hawaii has implemented a CWD surveillance program. An article in the October 24, 2003, edition of the Denver Post reported that hunting added $\$ 599$ million to the economy of the state of Colorado in 2002. The same article estimated the potential economic impact of CWD nationwide to be up to $\$ 100$ billion annually.

Chronic Wasting Disease in the Rocky Mountain region is concentrated in Northeast Colorado and Southeastern Wyoming. The disease has slowly been making its way across Colorado and has recently crossed the continental divide (Colorado, 2003). Incidents of the disease in Central and Western Colorado have been isolated thus far. Despite this spread and the potential biological, economic, and human health impacts, a statistical model with spatial components has yet to be presented.

There are several standard differential equations commonly used to model epidemics. One such is the Lotka-Volterra predator-prey model adapted to diseases as given in Renshaw (1991) and Hoppensteadt (1975). Previous efforts for modeling CWD have used variants of these (Gross and Miller, 2001; Hobbs et al., 2003). Models based on differential equations typically focus on individual interactions between deer, impacts of changes in the birth and death rates (Gross and Miller, 2001) and modeling the spread as a diffusion process (Hobbs et al., 2002). These authors use stochastic forward integration as the primary tool for understanding and exploring spatio-temporal dynamics. While these models h422 ave provided many insights into the behavior of the disease, they homogenize the affected region into a single unit and do not have a direct link between data and mathematical model.

Waller et al. (1997), Cressie and Mugglin (2000), and Mugglin et al. (2000) have successfully used a correlated Poisson model for the spatial spread of highly contagious human diseases such as influenza. These models capture the epidemic 
dynamics through use of a control term in the model that is turned "on" and "off". We use a model that is different from those presented in Waller et al. (1997) and Mugglin et al. (2000), because of fundamental differences in the data. Typically, large quantities of reliable data are available for building models of human diseases such as influenza. The models of Waller et al. (1997), Cressie and Mugglin (2000), and Mugglin et al. (2000) exploit large sample sizes to use Poisson approximations for Binomial distributions. On the other hand, it is extremely difficult and expensive to obtain disease prevalence data on a wildlife population. Thus, models for CWD need be to appropriate for small samples.

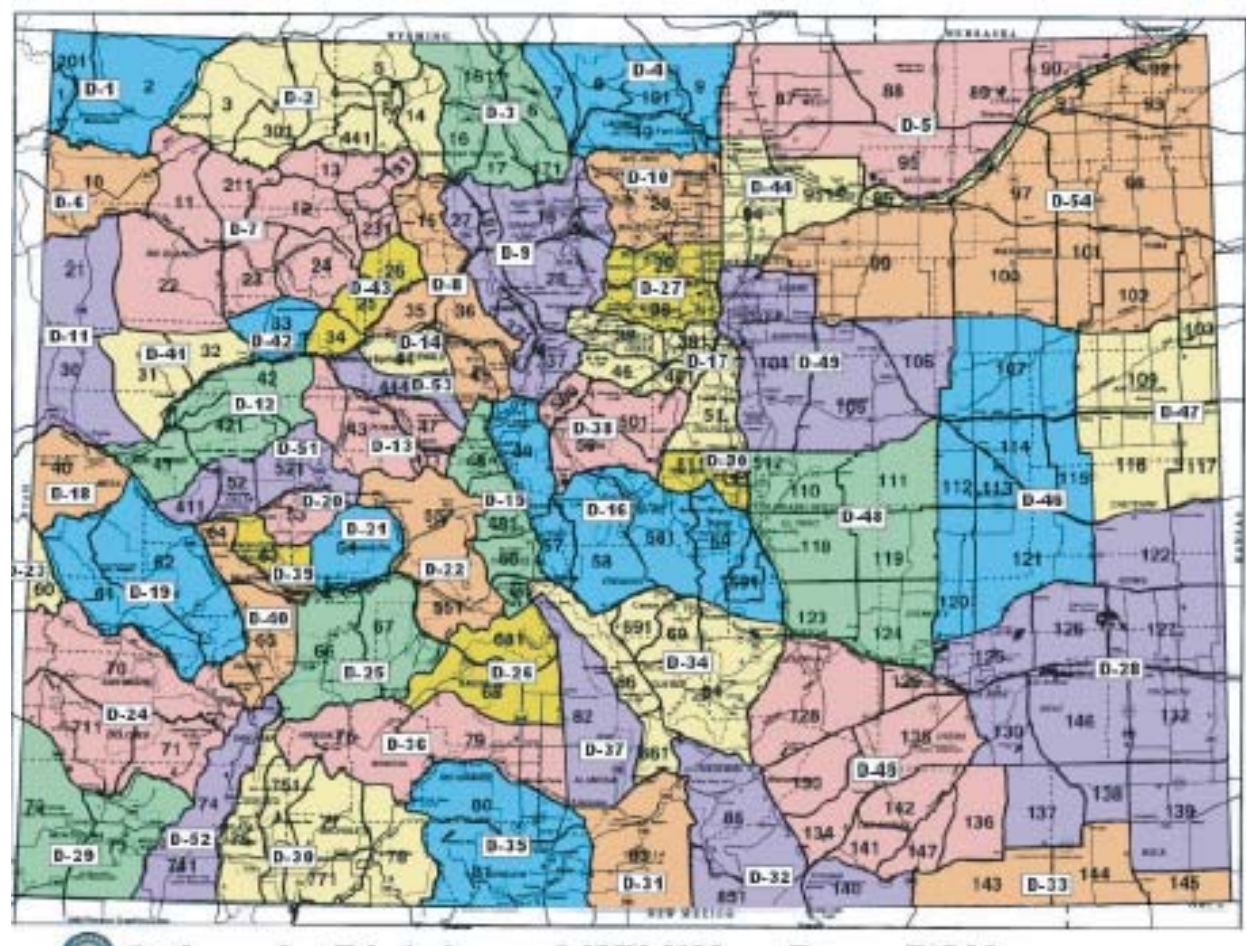

Golorado Division of Wildlife - Deer DAUs

Apeil as

Figure 1: Deer Analysis Units as defined by the Colorado Division of Wildlife. For reference, the Denver metro area is approximately centered where DAUs 17, 27 and 49 meet. The digits not in boxes represent smaller spatial units called game management units.

We propose a Bayesian hierarchical model for chronic wasting disease that directly integrates mathematical models related to those of Gross and Miller (2001), Hobbs et al. (2002) with a model for observations taken on CWD prevalence. Critical to the model is a difference equation approximation to traditional differential equation models of prevalence to model dynamics over time. We 
also include a mechanism that allows for spatial mixing of the disease between regions. We incorporate the available data through a Bayesian hierarchical framework, thus linking the data and mathematical model. This hierarchical approach integrates data and epidemic theory into a single model, is capable under the constraint of small data sets, and provides a mechanism for prediction and the study of data collection designs.

\section{Introduction}

Data for CWD is economically and ecologically expensive to obtain as the testing procedure, until recently, required sacrificing the animal, (Colorado, 2003, Hobbs et al., 2003). Prevalence data consists of the numbers of deer that are tested for CWD and the number of those tested that are found to have the disease (Miller et al., 2000) and Gross and Miller (2001).

Biologists divide the state of Colorado into 55 geographic regions called deer data analysis units (DAUs). The 55-th DAU was recently created from DAU 54 (north-east Colorado), and data from DAU-55 in this analysis is included with its mother DAU. The 54 DAUs are shown in Figure 1. The DAUs are defined from geographical and biological considerations and each is intended to represent a relatively homogeneous region. Data collected from the 1976-2002 hunting seasons has confirmed presence of CWD in thirteen DAUs. The data used in this study are courtesy the Colorado Division of Wildlife $(\mathrm{CDOW})^{1}$.

From 1976-1995, few deer (65) were tested, and those only from the endemic area, which consists of DAUs 4, 5, 10, 27, and 44. From 1996-2001, intense testing was done by the Colorado Division of Wildlife in this endemic area. The data consists of the number of deer tested in DAU $i$ and year $t\left(N_{i t}\right)$, and the number of those that test positive for $\operatorname{CWD}\left(M_{i t}\right)$. This includes data from both harvest (i.e., collected from hunters) and non-harvest sources. A majority of the data is harvest based. Harvest data may not always provide a glimpse of the population as a whole, since in some DAUs hunters are restricted to taking male animals only, while in others they may take animals of either sex, depending on the State's management plan. (Conner et al. 2000) notes differences in the harvest versus non-harvest based samples.

The 2002 and 2003 hunting seasons were different in that the CDOW did "no cost to hunter" testing on harvested animals from the endemic area, and offered testing at a minimal cost to hunters from all other areas Colorado (2003). In these years, CDOW required all deer and elk from the endemic area (northeastern Colorado) to be tested, and relied on voluntary submissions outside the area.

The difference in sampling mechanism is for areas where the disease is believed

\footnotetext{
${ }^{1}$ See http://www-math. cudenver. edu/ cjohns/CWDdata/
} 
to have "high" prevalence compared with areas where the disease is not yet known to have a significant presence puts an acute emphasis on the need for a model that can predict the movement of the disease. Specifically, in areas where prevalence exists, but at very low rates, it will take a significant amount of time for data evidence to be sufficient to require mandatory testing in areas that are currently thought to be disease free. The current sampling scheme that relies on voluntary submissions improves spatial coverage significantly, but may introduce selection bias.

\section{Spatio-Temporal Model}

Our approach is to construct a system of difference equations for prevalence that have qualitative temporal and spatial behavior appropriate for the current knowledge of CWD. Through a hierarchical Bayesian construction, we then use the data to estimate the unknown parameters that govern the difference equations. One fundamental aspect of our approach is the explicit modeling of spatial dynamics in the difference equations, rather than modeling the effect through correlation structure. In essence, we have a nonlinear state-space model with deterministic updates for the states.

We begin by forming a difference equation model for CWD, specifically requiring that it be flexible enough to allow different prevalences for each DAU. We take as a starting point differential equations that describe the growth and decay of populations and diseases Renshaw (1991). Of particular use are versions of the logistic growth curve. Hoppensteadt (1975) and Renshaw (1991) show that a differential equation for prevalence of a disease equivalent to the logistic model can be formed by a simple scaling. The basic model is defined by:

$$
\frac{d p}{d t}=\alpha^{\star} p(1-p)
$$

where $p$ is the prevalence of the disease.

For multiple DAUs we simply replicate equation (3.1) once for each DAU and collect into a vector. Letting $\mathbf{p}_{t}$ denote the vector of prevalences, one for each DAU, we extend the system to include spatial effects with:

$$
\frac{d \mathbf{p}_{t}}{d t}=\alpha^{\star} \mathbf{p}_{t} \odot\left(\mathbf{1}-\mathbf{p}_{t}\right)+Q \mathbf{p}_{t}
$$

where $\odot$ represents the Hadamard (element by element) product and $Q$ is a matrix such that the values $q_{i j} \in[0,1]$ represent the instantaneous net effect that the prevalence in DAU $j$ has on the prevalence of DAU $i$.

From historical observations and recent studies of CWD, Miller et al. (2000) and Gross and Miller (2001), it is known that CWD takes approximately 3 years 
from infection to the death of the animal. For a disease with an incubation period this long, it is reasonable to suppose that a sustainable level of the disease is possible. Furthermore, CWD has yet to show signs of elimination even in those areas where the disease has been present the longest, giving further support to this assumption. Another reasonable assumption is that if the disease is not present in a given area, i.e., if $p_{k t}=0$, then the proportion of deer that are infected will stay at zero, unless the disease is carried into the unit from some other unit, either by migration of infected deer or some other carrying agent. These two assumptions are evinced by the current understanding of the disease and provide support, through qualitative behavior, for choice of the logistic model (3.1) as a starting point.

The differential equation (3.2) is approximated by a first order Euler discretization of the form: $\mathbf{p}_{t+1}=\mathbf{p}_{t}+\alpha \mathbf{p}_{t} \odot\left(\delta \mathbf{1}-\mathbf{p}_{t}\right)+W \mathbf{p}_{t}$. Holding $\alpha^{\star}$ constant in this equation or (3.2) restricts the dynamics of the model by fixing the infection rate whereas letting $\alpha^{\star}$ be a function of $p$, gives some added flexibility to the model. Cressie and Mugglin (2000) and Mugglin et al. (2000) exploit this type of freedom in a model for influenza epidemics and explicitly introduce a parameter to turn the epidemic "on" and "off". We follow that lead and set $\alpha^{\star}=\alpha(\delta \mathbf{1}-\mathbf{p})$ where $\delta$ is scalar. With this modification, prevalence is increasing for levels below $\delta$, and decreasing for levels above $\delta$. If $\delta=0$, then the disease is killing off the infectives faster than it is creating new infectives and there will be an eventual burn out of the disease. For $\delta=1$, then the disease will eventually infect all the deer. When $0<\delta<1$, the model has a long-run stable proportion of infectives at level $\delta$. Thus the difference equations for our model are of the form:

$$
\mathbf{p}_{t+1}=\mathbf{p}_{t}+\alpha\left(\delta \mathbf{1}-\mathbf{p}_{t}\right) \odot \mathbf{p}_{t} \odot\left(\mathbf{1}-\mathbf{p}_{t}\right)+W \mathbf{p}_{t}
$$

where $W$ is a spatial mixing matrix that represents deer migration. We note here that migration changes prevalence in that a single infected deer migrating from one DAU to another changes the makeup of prevalence for both the "from" and "to" DAUs. Thus the change in prevalence for a given DAU consists of a mixture of the internal (within DAU) dynamic processes modeled by the logistic equations, (the first term on the right hand side of (3.3)), and the external contributions from migration to and from neighboring DAUs, (the $W \mathbf{p}_{t}$ term in (3.3)). Including the migration matrix $W$ does not imply, nor remove the possibility, that deer are the transmission method for the disease in the model.

In constructing the spatial mixing matrix, $W$, we assume that the disease can only migrate between adjoining DAUs, so that if $\mathrm{DAU}_{i}$ and $\mathrm{DAU}_{j}$ are neighbors, and $\mathrm{DAU}_{j}$ and $\mathrm{DAU}_{k}$ are neighbors, but $\mathrm{DAU}_{i}$ and $\mathrm{DAU}_{k}$ are not, then in a single time step (one year for our case) migration can occur between $i$ and $j$, and between $j$ and $k$, but not between $i$ and $k$. Given what is known about 
the behavior of deer in the wild and the spread of CWD in Colorado, this is a reasonable assumption.

Table 1: Neighborhood relationships between DAUs. Numbers refer to DAU number in Figure 1.

\begin{tabular}{|c|c|c|c|c|c|}
\hline DAU & $\begin{array}{l}\text { Near } \\
\text { Neighbors }\end{array}$ & $\begin{array}{l}\text { Secondary } \\
\text { Neighbors }\end{array}$ & DAU & $\begin{array}{l}\text { Near } \\
\text { Neighbors }\end{array}$ & $\begin{array}{l}\text { Secondary } \\
\text { Neighbors }\end{array}$ \\
\hline 1 & 2,6 & 7 & 28 & $33,45,46,47$ & 48 \\
\hline 2 & $1,3,4$ & 8,9 & 29 & 24,52 & - \\
\hline 3 & $2,4,9$ & 8,10 & 30 & $35,36,52$ & 25 \\
\hline 4 & $2,3,5,10$ & 9,44 & 31 & $32,35,36$ & 37 \\
\hline 5 & $4,44,54$ & - & 32 & $31,34,45$ & 33 \\
\hline 6 & $1,7,11$ & - & 33 & 28,45 & 32 \\
\hline 7 & $6,8,11,41,42,43$ & 1 & 34 & $16,32,37,45$ & $15,26,48$ \\
\hline 8 & $7,9,14,43$ & $2,3,15,16,53$ & 35 & $30,31,36$ & 37 \\
\hline 9 & $3,8,10,17,27$ & $2,4,16,38$ & 36 & $25,26,30,31,35,37$ & - \\
\hline 10 & $4,9,27,44$ & 3 & 37 & $26,34,36$ & $15,31,35$ \\
\hline 11 & $6,7,18,41$ & 12 & 38 & 16,17 & 9,50 \\
\hline 12 & $13,41,51$ & $11,18,19,42,43$ & 39 & $20,21,40$ & 25,51 \\
\hline 13 & $12,15,22,51$ & 42,43 & 40 & $19,25,39$ & 24,51 \\
\hline 14 & $8,43,53$ & - & 41 & $7,11,12$ & 42 \\
\hline 15 & $13,16,22$ & $8,26,34,37,53$ & 42 & 7,43 & $12,13,41$ \\
\hline 16 & $15,34,38,48,50$ & $8,9,45$ & 43 & $7,8,14,42$ & $12,13,53$ \\
\hline 17 & $9,38,44,49,50$ & 48 & 44 & $5,10,17,54$ & 4,27 \\
\hline 18 & 11,19 & 12,23 & 45 & $28,32,33,34,48$ & 16,46 \\
\hline 19 & $18,23,24,40$ & 12,51 & 46 & $28,47,48,49,54$ & 45 \\
\hline 20 & $21,39,51$ & 22 & 47 & $28,46,54$ & - \\
\hline 21 & $20,22,25,39$ & $\overline{-}$ & 48 & $16,45,46,49,50$ & $17,28,34$ \\
\hline 22 & $13,15,21,25,26$ & 20 & 49 & $17,27,46,48,54$ & 50 \\
\hline 23 & 19 & 18,24 & 50 & $16,17,48$ & 38,49 \\
\hline 24 & $19,29,52$ & 23,40 & 51 & $12,13,20$ & $19,39,40$ \\
\hline 25 & $21,22,26,36,40$ & $30,39,52$ & 52 & $24,29,30$ & 25 \\
\hline 26 & $22,25,36,37$ & 15,34 & 53 & 14 & $8,15,43$ \\
\hline 27 & $9,10,49$ & 44 & 54 & $5,44,46,47,49$ & - \\
\hline
\end{tabular}

For parsimony, we fix $W_{i j}=\gamma$ if $\mathrm{DAU}_{i}$ and $\mathrm{DAU}_{j}$ are neighbors with a "significant" proportion of boundary that touch. If $\mathrm{DAU}_{i}$ and $\mathrm{DAU}_{j}$ have less of boundary that touch, or are almost touching, then $W_{i j}=\omega<\gamma$. For DAUs that are separated, $W_{i j}=0$. The diagonal values of $W$ are chosen to make the 
row sums equal to 1 . Thus, the $W_{i j}$ can be loosely interpreted as the average proportion of deer that migrate from $\mathrm{DAU}_{i}$ to $\mathrm{DAU}_{j}$ each year. Table 1 shows the ad hoc relationships between the DAUs that we used.

The observation at $\mathrm{DAU}_{i}$ at year $t$, denoted by $Y_{i t}$, is modeled as one of two binomial distributions

$$
\begin{aligned}
Y_{i t} & \sim B\left(N_{i t}, p_{i t}\right) & & \text { for } N_{i t}>\mathcal{C} \\
& \sim B\left(N_{i t}, \pi\right) & & \text { for } N_{i t} \leq \mathcal{C} .
\end{aligned}
$$

depending on some cutoff $\mathcal{C}$ and where $N_{i t}$ is the number of tests completed for $\mathrm{DAU}_{i}$ at year $t$ and $p_{i t}$ is the $i^{\text {th }}$ component of $\mathbf{p}_{t}$. This duality is required because of the different sampling schemes for different stages of the knowledge of disease prevalence. The first distribution corresponds to time-periods where a DAU has a large number of deer tested for the disease. In these cases, the Division of Wildlife knows that the disease is present and wishes to protect hunters from eating infected animals. On the other hand, combinations of DAUs and years when the number of tested carcasses is low correspond with the CDOW searching for the disease in an area where it has not yet been found or confirmed problematic. This data comes from animals found dead or ill, and hunters that are willing to pay for the test. This search for the disease adds bias which we explicitly include in the model with the parameter $\pi$ and cutoff $\mathcal{C}$.

We define $\eta=(\alpha, \delta, \gamma, \omega)$ as the vector of parameters for the update equation (3.3). Given $\eta$ and a starting vector of prevalences, $\mathbf{p}_{0}$, the updates of $\mathbf{p}_{t}$ are fixed and the likelihood is given by,

$$
\begin{aligned}
\mathcal{L}\left(\mathbf{Y} \mid \mathbf{p}_{\mathbf{0}}, \eta, \pi, \mathcal{C}\right)= & \prod_{N_{i t}>0}\left[p_{i t}^{Y_{i t}}\left(1-p_{i t}\right)^{\left(N_{i t}-Y_{i t}\right)}\right]^{1_{\left\{N_{i t}>\mathcal{C}\right\}}} \\
& \times\left[\pi^{Y_{i t}}(1-\pi)^{\left(N_{i t}-Y_{i t}\right)}\right]^{1_{\left\{N_{i t} \leq \mathcal{C}\right\}}}
\end{aligned}
$$

where $1_{\mathcal{A}}$ is 1 if condition $\mathcal{A}$ is true and zero otherwise. We assume that the value of $p_{i, t+1}$ in (3.5) depends on $p_{i t}$ only through equation (3.3). The value $\mathcal{C}=3$ provides a reasonable fit to the data (see Section 4). The posterior distribution of parameters is proportional to

$$
\mathcal{P}\left(\mathbf{p}_{0}, \eta, \pi\right) \propto \mathcal{L}\left(\mathbf{Y} \mid \mathbf{p}_{\mathbf{0}}, \eta, \pi, \mathcal{C}\right) \amalg\left(\mathbf{p}_{\prime}\right) \amalg(\eta) \amalg(\pi)
$$

where $q(\cdot)$ denotes a prior distribution.

This model has qualitative characteristics, through the deterministic differential equations, that are consistent with current knowledge of CWD and is flexible enough to accommodate spatial mixing of prevalences due to deer migration and possibly other factors. Formalizing the model with a Bayesian hierarchy gives 
the added benefit of methods for comparing different models, testing whether or not the disease is spreading in space (if $\gamma>0$ ) and whether or not there disease can coexist with the mule deer population (if $0<\delta<1$ ). The Bayesian hierarchy also provides a way to check sampling schemes that will maximize information from data collected in years to come.

Prior distributions for $\alpha, \delta$ and $\pi$ were all independent Beta distributions. Specifically we chose a uniform prior for $\alpha$, $\operatorname{Beta}(8,82)$ for $\delta$ and symmetric $\operatorname{Beta}(1.5,1.5)$ for $\pi$. For the migration parameters $\gamma$ and $\omega$, the distribution is proportional to the product of two Beta distributions, $\operatorname{Beta}(1,50)$ and $\operatorname{Beta}(1,100)$ respectively, subject to the constraints that $\gamma>\omega$ and that $\max _{i}\left\{\sum_{j \neq i} W_{i j}\right\}<1$ Prior distributions for $\mathbf{p}_{0}$ were either $\operatorname{Beta}(1,200)$ or point mass at zero.

We formulated several versions of (3.3) with the goal of letting Bayes factors (see equation (4.1)) guide our choice of model. The first set of models assume that only a single DAU is infected at the $0^{\text {th }}$ time step corresponding with 1975 , and all others have an initial value of zero. This is accomplished formally by letting the prior distribution for $p_{i 0}$ be a point mass at zero for all DAUs except the DAU of interest. The second set of models, follows in a stepwise-forward manner and assume that two DAUs have positive, distinct prevalences at time 0 , while all other DAUs start with zero prevalence. The pattern continues adding one DAU at at time. To reduce the search space, we focused on models for which DAUs with the positive prevalence in 2002 had the ability to enter the model with postive prevalence in 1975 . We also explicitly ran models with $\omega>0$ and $\omega=0$ in order to test whether or not the second order migration term affects the model.

\section{Results}

We first find a reasonable cutoff value, $\mathcal{C}$, for the likelihood (3.5). The joint posterior distribution (3.6) can be factored so that the posterior of $\pi$ given all the data is independent of the posterior of $\eta, \mathbf{p}_{0}$. Thus, doing a search under any model for the best level of $\mathcal{C}$ with a symmetric prior for $\pi$ will yield a cutoff level as close to $50 \%$ of observations that are diseased and $50 \%$ that are not. This fact is obvious when considering that, for given $n$, the binomial distribution with $p=.5$ accounts for more data variability than any other model. Thus, we found that $\mathcal{C}=3$. In DAU-years where there are 4 or more carcasses tested for CWD, the modeled prevalence will be from the updates given in (3.3), otherwise, we assume that the proportion of deer that are tested and that actually have the disease is $\pi$.

We compared the different models mentioned in Section 3 using Bayes factors. Bayes factors are ratios of the distributions for the data, conditioned upon the 

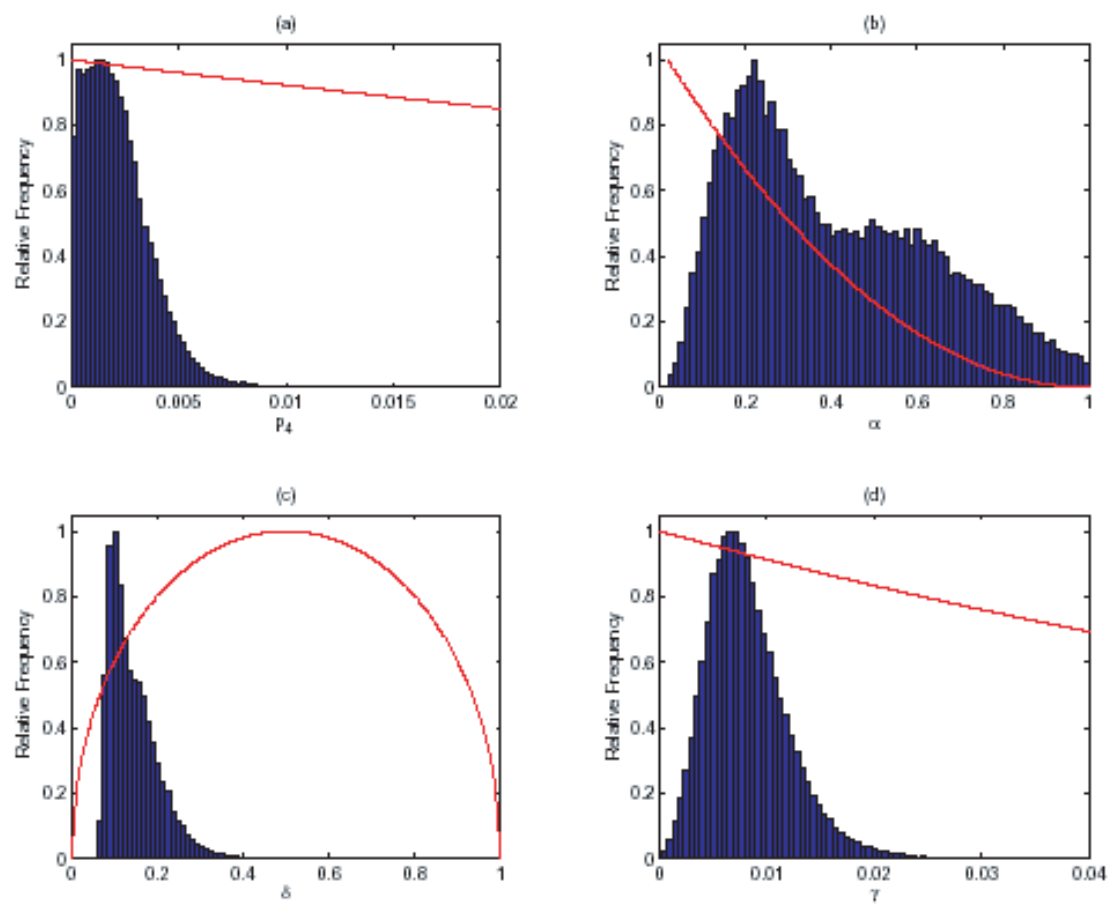

Figure 2: Comparisons of the posterior sample with prior distributions for parameters (a) $p_{10_{0}} 10$, (b) $\alpha$ the acceleration parameter, (c) $\delta$ the long run prevalence and (d) $\gamma$, the average propensity to migrate. The prior and histogram approximation of the posterior have been scaled to have maximum value of one in order to highlight the difference between the two distributions.

model:

$$
B F(i ; j)=\frac{\int \mathcal{L}\left(\mathbf{Y} \mid \eta, \mathbf{p}_{\mathbf{0}}, \pi, \mathcal{M}_{\mathbf{i}}\right) \mathbf{q}\left(\mathbf{p}_{\mathbf{0}}\right) \mathbf{q}(\eta) \mathbf{q}(\pi) \mathbf{d} \mathbf{p}_{\mathbf{0}} \mathbf{d} \eta \mathbf{d} \pi}{\int \mathcal{L}\left(\mathbf{Y} \mid \eta, \mathbf{p}_{\mathbf{0}}, \pi, \mathcal{M}_{\mathbf{j}}\right) \mathbf{q}\left(\mathbf{p}_{\mathbf{0}}\right) \mathbf{q}(\eta) \mathbf{q}(\pi) \mathbf{d p}_{\mathbf{0}} \mathbf{d} \eta \mathbf{d} \pi}
$$

where $\mathcal{M}_{i}$ implies that the integration is with respect to the prior and likelihood under model $i$. We approximate the numerators and denominators of the Bayes factors via a random sample of the prior distributions implied by the models $\mathcal{M}_{i}$ and $\mathcal{M}_{j}$. For $k=1, \ldots K$, draw the appropriate collection of parameters, denoted by $\theta_{i k}$, from the prior distribution imposed by $\mathcal{M}_{i}$. Let $\ell_{i k}$ denote the evaluation of the log-likelihood implied by (3.5) evaluated at $\theta_{i k}$ and $w_{i k}=\exp \left(\ell_{i k}-\max _{k}\left\{\ell_{i k}\right\}\right)$ and $\bar{w}_{i}=\sum_{k} w_{i k} / K$. Then $\hat{B F}(i ; j)=$ $\exp \left\{\ell_{i \max }-\ell_{j \max }\right\} \bar{w}_{i} \cdot / \bar{w}_{j}$. The Bayes factor gives a weighting of the relative skill of model $i$ in fitting the data as compared with model $j$, and is related to a likelihood ratio test. Under this criterion, the model with $p_{4_{0}}, p_{5_{0}}, p_{10_{0}}>0$, all other DAUs having zero starting values, and $\omega=0$ is as or more effective than other 

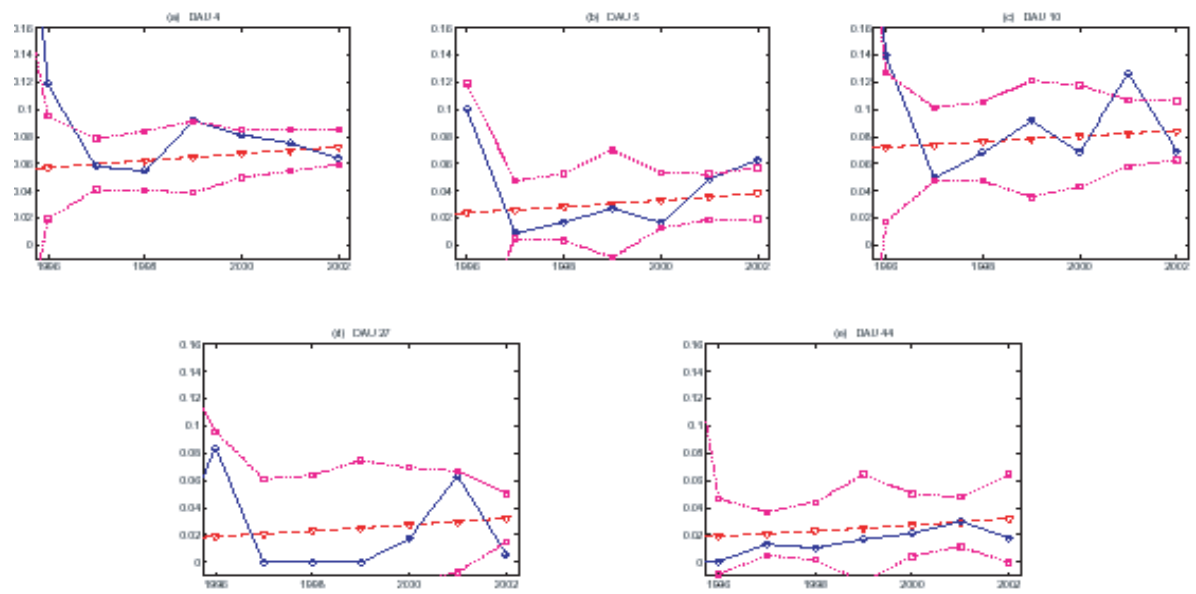

Figure 3: Fitted values and corresponding frequentist confidence intervals for the proportion of infected deer, based on the component wise median of the posterior sample. Depicted are (a) DAU 4, (b) DAU 5, (c) DAU 10, (d) DAU 27 and (e) DAU 44. Observed prevalences are denoted with o, 95\% confidence intervals are denoted by $\square$ and the predicted value is denoted by $\nabla$.

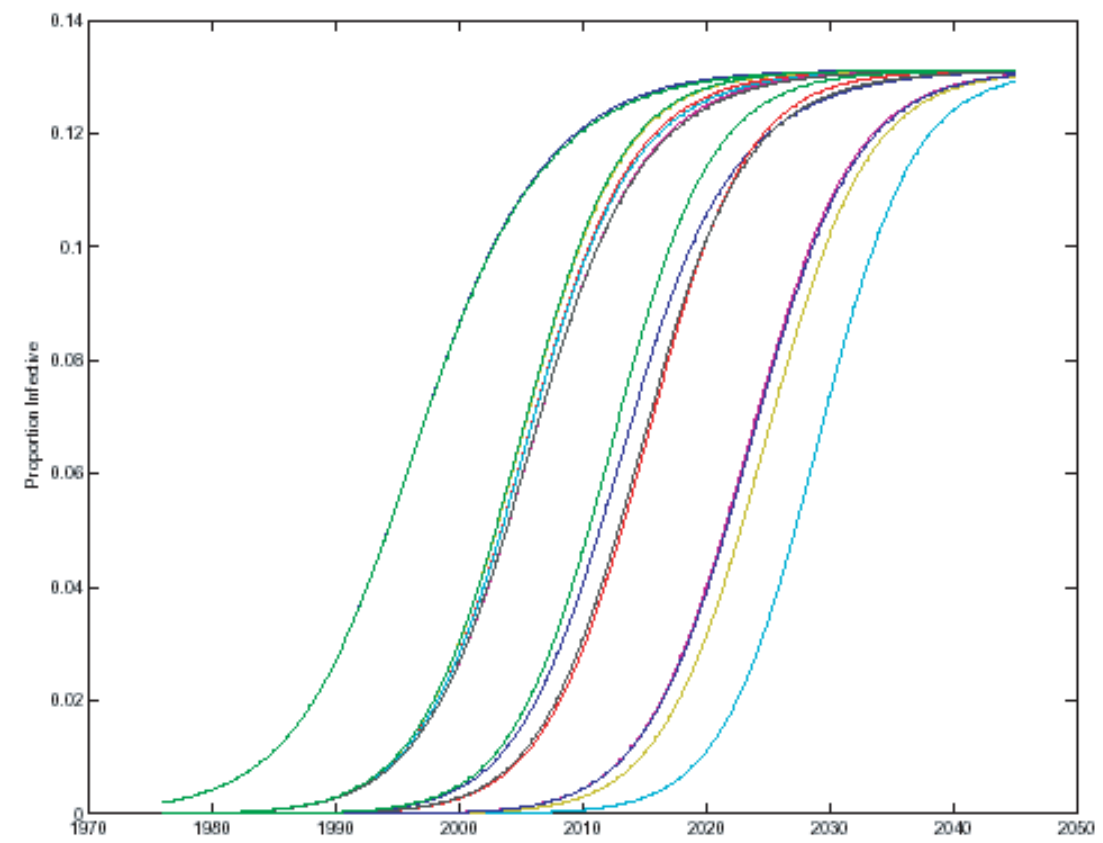

Figure 4: Predicted prevalences, for all DAUs based on the median curve based on the posterior random sample. 
models with the same number or more parameters. The estimated Bayes factor was 1.18 when comparing this model with the similar model that also included non-zero initial values for DAUs 3, 17, and 9. The next smallest Bayes factor (3.55) was for the model that forced $p_{5_{0}}=0$.
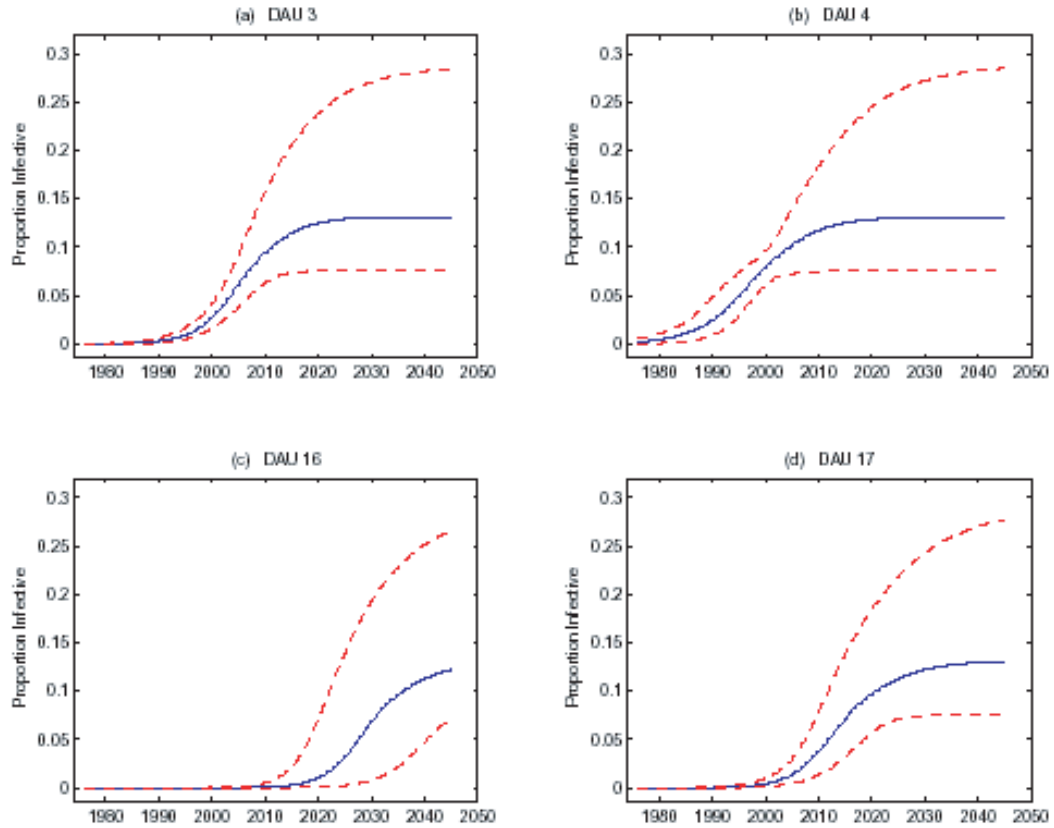

Figure 5: Solid line is the median track for prevalence based on the posterior sample $(\mathrm{N}=25,000)$. Dashed lines represent pointwise $95 \%$ credible intervals.

As the posterior distribution for this model depends on many nonlinear relationships, we characterized its behavior using a random sample of size 25,000 drawn via an acceptance sampler. Scaled histograms of the variables $p_{10_{0}}, p_{4_{0}}, p_{5_{0}}$, $\alpha, \delta$, and $\gamma$ from the posterior sample are given in Figure 2. For comparison, the prior distribution is shown with a solid line. The prior and histogram approximation of the posterior have been scaled to to highlight the difference between the two distributions giving some indication of the ignorance in the priors and the strength of the data in the model.

Figure 3 shows the fitted prevalences, based on the median of posterior sample (depicted by dashed line and $\nabla$,) with corresponding $95 \%$ frequentist confidence intervals for the prevalences (dotted line, $\square$ ). The figure shows that the model provides a reasonable fit to the data (solid line, o). The five DAUs shown here are those in the endemic are, and the data corresponding to the five panels are 
primarily responsible for the model form as they carry much of the information in the likelihood and hence posterior distribution.

Figure 4 shows the predicted prevalence curve for the DAUs based on the year by year medians of the prevalence tracks based on the posterior sample. The curves maintain the sigmoidal shape given by the logistic equation, increasing with time toward the long-term stable state $\delta=0.149$. Those DAUs that were first infected approach $\delta$ first. Because the spatial mixing term $\gamma$ is greater than zero, and because every DAU is connected with at least one other DAU via the relationships in Table 1, all DAUs eventually become infected, and thus eventually approach the stable state.

Figure 5 shows the medians (solid line) and 95\% credible intervals (dashed lines) for the prevalence tracks over time for the five endemic DAUs. The intervals are narrow in the period where the data is strong (1996-2002) and grow wider as we project further into the future. While the model is a greatly simplified caricature of the actual dynamic process, it provides both a reasonable fit to the data and reasonable predictions for future prevalence.

The Bayesian hierarchy allowed us to test and compare various sampling schemes for 2003. For the comparison, we assumed that approximately 10,000 total mule deer tests for CWD could be afforded (just slightly above the 9,810 tests conducted in 2002.) To build sampling schemes, DAUs were classified into one of four groups; (1) the endemic area, DAUs where the disease is well established $(4,5,10,27,44) ;(2)$ the new area, DAUs where the disease was found in $2002(2,3,6,7,8,9,12,17)$; (3) neighbors, DAUs that are not in group 1 or 2 but are "neighbors" (as defined in Table 1) to one or more DAUs in groups 1 or 2 (11 DAUs in this group); and (4) others, DAUs not near an area known to have the disease (30 DAUs in this group). Relative weights were given to each group according to the digits in the first column of Table 2, and sample sizes for each DAU were calculated based on those weights and group membership.

Let $\beta=\left(p_{0_{10}}, p_{0_{4}}, p_{0_{5}}, \alpha, \delta, \gamma\right)$ denote the parameters of scientific interest in the model and $\beta_{j}^{\star}$ denote the $j$-th vector in the posterior sample. For each sampling design, 500 random draws from the posterior were made and for each random draw the model was run forward to 2003. A random sample ( $\left.\mathbf{Y}_{k}^{\star} 2003\right)$ of binomial random variables (see equation (3.4)) for year 2003 was generated assuming the model fit implied by the random draw from the posterior sample and the sample size imposed by the design. The conditional expectation, $E\left(\beta \mid \mathbf{Y}_{k, 2003}^{\star}\right)$ was estimated by $E\left(\widehat{\beta \mid Y_{k, 2003}^{\star}}\right)_{k}=\sum_{j=1}^{5000} \beta_{j}^{\star} w_{j}$ with $\beta_{j}^{\star}$ a random draw from the posterior sample and $w_{j}$ proportional to the evaluated conditional distribution of $\mathbf{Y}_{k}^{\star} 2003$ given $\beta_{j}^{\star}$. We compared the sample variances of the 500 estimated conditional means to compare the 13 sampling schemes.

In particular, we compared estimated generalized covariance $(\operatorname{det}(\hat{\Sigma}))$ and 
estimated total variance $(\operatorname{tr}(\hat{\Sigma}))$ for the 13 designs in Table 2 . Here $\hat{\Sigma}$ denotes the estimated covariance matrix, and $\operatorname{det}()$ denotes the determinant. The designs that put most emphasis on the areas with least data to date yields the best improvement in terms of generalized variance and total variance. Further, for each element of $\beta$, the first four designs in the table have the same order and relative standing based on the estimated conditional variance of the parameter.

Table 2: Sample scheme weights with corresponding sample sizes for each group and total sample size for 13 theoretical sampling schemes. Numbers in the logratios column represent the log of the ratio of estimated generalized covariance $(|\hat{\Sigma}|)$ of the estimated conditional means under the sampling scheme compared with scheme 1144. Digits in the scheme column represent the relative weights of the sample sizes for the four groups.

\begin{tabular}{ccccccc}
\hline Scheme & Group 1 & Group 2 & Group 3 & Group 4 & N & log-ratios \\
\hline 1144 & 56 & 56 & 224 & 224 & 9,912 & 0,0 \\
1142 & 105 & 105 & 420 & 210 & 9,945 & 1.49 \\
1122 & 105 & 105 & 210 & 210 & 9,975 & 2.11 \\
1141 & 115 & 115 & 460 & 115 & 10,005 & 2.99 \\
1441 & 90 & 360 & 360 & 90 & 9,990 & 3.99 \\
1241 & 85 & 170 & 340 & 85 & 9,975 & 4.06 \\
1111 & 185 & 185 & 185 & 185 & 9,990 & 4.24 \\
1221 & 137 & 234 & 234 & 137 & 10,001 & 4.55 \\
1211 & 161 & 322 & 161 & 161 & 9,982 & 5.10 \\
2441 & 172 & 344 & 344 & 86 & 9,976 & 5.50 \\
2211 & 248 & 248 & 149 & 149 & 9,983 & 6.24 \\
2111 & 338 & 169 & 169 & 169 & 9,971 & 7.12 \\
4221 & 456 & 228 & 228 & 114 & 10,032 & 7.34 \\
\hline
\end{tabular}

\section{Discussion}

While prevalence is an important aspect of studying CWD, it is not the only one. In many cases of equal importance in understanding the disease is the number of deer that are in a given region and the ability of the disease to "burn out," coexist, or kill off the host. Although we have not explicitly modeled the interplay between total population size and prevalence, there are many differential equations models designed for such interactions but such models that include the interaction of population size with prevalence are beyond the scope of this paper. Notwithstanding, the model presented here does give some evidence that the disease and the deer population can coexist. 
The posterior distribution on the long run disease prevalence $\delta$ has median and mode at approximately 0.148 . The corresponding estimated $95 \%$ credible interval is $(11.2 \%, 20.4 \%)$. This gives some evidence of a non-zero long-run sustainable level for the disease and that it is unlikely to disappear from the deer population. Thus, carefully crafted strategies are of critical importance in order to manage CWD.

There are three sources of uncertainty that contribute to the width of the intervals in Figure 5. First is Monte Carlo error as the lines are based on a random sample rather than explicitly calculated integrals of the posterior distribution. This error was mitigated by using a random sample of size 25,000. Secondly is uncertainty from the prior distributions propagated through the model. This variability can be reduced by larger sample sizes in the future as the data will swamp the prior. While the model presented here is sensible and stands on firm mathematical underpinnings, it is not likely to be an exact representation of the complex biological process controlling CWD. The deterministic updates for prevalence are quite strict and may not accurately reflect the true nature of disease spread. Features of the model contain structures that reflect the current scientific knowledge of CWD. From what is currently known, the steady increase to a sustainable level is reasonable over the given time frame.

Pairwise comparisons of the marginal posterior distributions indicate that several of the variables are highly correlated. The plot of $\alpha$ versus $\delta$ (not shown) from the posterior sample shows that nearly all of the simulated values occur on a curvilinear ridge of high probability. Comparisons among $\alpha, \delta$, and $\gamma$ show that all these variables are all highly correlated with each other. The high correlation between these variables makes it difficult to construct an efficient Markov chain sampler for the posterior. In this application, the problem was circumvented using an acceptance/rejection sampler, facilitated by the parsimony of the model. An efficient Markov Chain sampler would be a valuable addition so that related, more complicated models can be applied to this or other diseases and data situations. Efficient MCMC sampling is beyond the scope of this paper and reserved for future work.

As more is learned about the disease, the model can be modified to include the added information. In particular knowledge of how the disease is spread could be used to improve the disease migration effects modeled by elements of matrix $W$ in equation (3.3). A change in the form of $W$ with current data may have some effect on the estimation of $\delta$, but these differences are likely to be quantitative rather than qualitative. Likewise, as more is learned about transmission mechanism, modifications to the form of acceleration, currently, $\alpha(\delta \mathbf{1}-\mathbf{p})$, can be considered to reflect that knowledge. In particular, it is possible to let the acceleration parameter, $\alpha$, depend on DAU and covariates, such as deer density, range type 
and condition, and incorporated into the model via a logistic regression equation for the level of $\alpha$ as a function of the covariates. Recent efforts by the Colorado Division of Wildlife, such as the recent moratorium on transport of "domestic" deer and elk from ranches or the increase in hunting season lengths and number of licenses issued in endemic areas, may have an impact on the spread of the disease. The effects of these kinds of policies could also be incorporated into the model through modifications to the accelleration/decelleration parameter, $\alpha$.

We have made some accommodations in the model to account for different sampling schemes that are used based on the then current level of knowledge regarding disease prevalence. Still, these accommodations are ad hoc and only moderately reflect the true nature of the sampling mechanism. Notwithstanding, the modification provides the model sufficient flexibility to accurately describe the changing prevalences. This modification, in some sense, eliminates data from the spatio-temporal model as the posterior of $\pi$ given all the data is independent of the other parameters. In essence, data that are used to improve knowledge regarding $\pi$ cannot be used to increase information about the other parameters.

Another possible avenue of future research is to determine the impact different control strategies imposed by a state wildlife division might have on the posterior distribution of $\delta$, and whether the controls could be used to lower the long run prevalence to a level close to zero. The Bayesian hierarchy allows for different schemes to be tested via computational simulation prior to implementation. In particular, the sampling designs in Section 4 suggest that the CDOW should increase testing of deer harvested in non-endemic areas to gain the most information about the transmission of the disease. This corresponds with conventional wisdom that one learns more by asking questions for which the answer is unknown than one learns by asking questions for which the answer is known. Part of the effect may be due to the binomial assumption and the relationship between the probability (prevalence) and variance of observations. Namely, the variance

of binomial distributions is smaller for distributions with smaller probabilities of "success."

\section{Acknowledgments}

The authors thank the Colorado Division of Wildlife for providing the data of this paper and permission to use Figure 1.

\section{References}

Conner, M., McCarty, C., and Miller, M. (2000). Detection of bias in harvest-based estimates of chronic wasting disease prevalence in mule deer. Journal of Wildlife Diseases 36, 661-699. 
Cressie, N. and Mugglin, A. (2000). Spatio-temporal hierarchical modeling of an infectious disease from (Simulated) count data. COMPSTAT: Proceedings in Computational Statistics. Physica-Verlag.

Gross, J. and Miller, M. (2001). Chronic wasting disease in mule deer: Disease dynamics and control. Journal of Wildlife Management 65, 205-215.

Hobbs, N., Miller, M., Williams, E., Burnham, K., Reich, R., Thomas, J., and Theobald, D. (2002). Spatial and temporal dynamics of prion disease in wildlife: Responses to changing land use. Natural Resource Ecology Laboratory 2002 progress report, Colorado State University, Fort Collins, CO.

Hobbs, N., Miller, M., Williams, E., Burnham, K.. Reich, R., Thomas, J., and Theobald, D. (2003). Spatial and temporal dynamics of prion disease in wildlife: Responses to changing land use. Natural Resource Ecology Laboratory 2003 progress report, Colorado State University, Fort Collins, CO.

Hoppensteadt, F. (1975). Mathematical Theories of Populations: Demographics, Genetics, and Epidemics. CBMS-NSF Regional Conference Series in Applied Mathematics. Society for Industrial and Applied Mathematics.

Miller, M., Williams, E., McCarty, C., Spraker, T, Kreeger, T, Larsen, C., and Thorne, E. (2000). Epizootiology of chronic wasting disease in free-ranging cervids in Colorado and Wyoming. Journal of Wildlife Diseases 36, 676-690.

Mugglin, A., Cressie, N., and Gemmell, I. (2000). Hierarchical statistical modeling of influenza-epidemic dynamics in space and time. Department of Statistics preprint no. 662, Ohio State University, Columbus, $\mathrm{OH}$.

Renshaw, E. (1991). Modeling Biological Populations in Space and Time. Cambridge Studies in Mathematical Biology. Cambridge University Press.

Waller, L, Carlin, B, Xia, H, and Gelfand, A. (1997). Hierarchical spatio-temporal mapping of disease rates. Journal of the American Statistical Association 92, 607-617.

Received March 20, 2004; accepted September 27, 2004.

Craig J. Johns

Mathematics Department

University of Colorado

Denver, CO, 80217-3364, USA

cjohns@math.cudenver.edu

Christopher H. Mehl

Mathematician

United States Department of Defense

Bolling AFB, Building 6000, B6-961

Washington D.C., 20340-5100

USA 